\title{
Drugging homologous recombination: back to the future
}

\author{
Hannah Sanford-Crane ${ }^{1}$, Tanja Pejovic ${ }^{2}$ \& Xiangshu Xiao*,1 \\ ${ }^{1}$ Program in Chemical Biology, Department of Physiology \& Pharmacology, Oregon Health \& Science University, 3181 SW Sam \\ Jackson Park Rd, Portland, OR 97239, USA \\ ${ }^{2}$ Department of Obstetrics \& Gynecology, Oregon Health \& Science University, 3181 SW Sam Jackson Park Rd, Portland, OR \\ 97239, USA \\ *Author for correspondence: Tel.: +1 503494 4748; xiaoxi@ohsu.edu
}

\begin{abstract}
'In conclusion, a better fundamental understanding of the regulation of the HR pathway and recent technology developments in designing inhibitors should facilitate rapid development of novel HR inhibitors as potential novel cancer therapies"
\end{abstract}

First draft submitted: 28 March 2018; Accepted for publication: 4 April 2018; Published online: 2 May 2018

\section{Keywords: cancer • DNA repair • homologous recombination • inhibitor • resistance}

If a person is lucky to live long enough, he/she will also have a significantly increased likelihood of developing a cancer. Cancer is caused by accumulation of gene mutations over time. Genomic instability is one of the two key enabling characteristics of cancer cells [1], first enabling initial tumorigenesis and later drug resistance. Genomic stability is critical to maintain cellular homeostasis and cellular identity. However, cellular DNA is constantly under the attack from both endogenous and exogenous threats, creating lesions. These lesions, particularly the DNA double-strand breaks (DSBs) are lethal to the cells. They must be repaired for the cells to survive and proliferate. There are two major pathways to repair the DSBs in mammalian cells. One is error-free homologous recombination repair (HR) and the other one is error-prone nonhomologous end-joining repair [2]. During HR, a homologous sister chromatin is used as a template to repair the breaks. In nonhomologous end-joining, on the other hand, the broken DNA ends are simply ligated together without a need for a homologous template, often leading to some deletions of the original DNA sequences. It is the HR process that maintains the integrity of our cellular genome.

\section{Reversing resistance}

While genomic instability is a hallmark of cancer cells, the genome cannot be too unstable. After passing a certain intolerable threshold, the cancer cells will die from excessive DSBs or genomic changes. This creates a unique opportunity to develop novel cancer therapies by targeting the HR pathway. The viability of targeting HR repair for anti-cancer therapeutics is supported by the recent US FDA approval of three different PARP inhibitors (olaparib, rucaparib and niraparib) for high-grade serous ovarian cancer (HGSOC). These PARP inhibitors are mostly effective in patients with preexisting somatic or germline $B R C A$ mutations through a process called synthetic lethality [3]. BRCA1 and BRCA2 are two distinct breast and ovarian cancer-associated tumor suppressor genes. They are essential for instigating the HR repair pathway in collaboration with the nuclease MRN (Mre11-Rad50-NBS1) complex [2]. Mutations in BRCA genes result in deficient HR repair activity, a process which cancer cells are particularly reliant on due to high level of replication stress [4]. In the case of HGSOC, carriers of $B R C A$, which account for only approximately $30 \%$ of the patients are more sensitive to existing drugs including platinum salts and PARP inhibitors present significantly better prognosis than noncarriers. Even these BRCA carriers eventually develop resistance to the PARP inhibitors or platinum drugs. Although the mechanisms underlying the resistance are incompletely understood, restoration of HR function is a major cause [5,6]. Therefore, pharmacological strategies to drug the HR pathway can potentially expand the utility of existing PARP inhibitors and platinum drugs by priming the non-BRCA carriers and reversing the resistance mechanisms seen in the clinic with PARP inhibitors and platinum drugs. Practically, because HGSOC patients are routinely screened to assess $B R C A$ mutations, this type of therapy can be easily incorporated into precision oncology therapy arena. If $B R C A$ mutations (germline 
or somatic) are identified, the patients can be treated with existing PARP inhibitors or platinum salts. Once these patients develop drug resistance through restoration of HR pathway, an HR inhibitor can be added to the patient's treatment regimen. If $B R C A$ mutations are not found, the patients can be treated with an HR inhibitor alone or in combination with existing PARP inhibitors or platinum salts.

\section{Targeting the homologous recombination repair pathway}

The HR pathway involves a hierarchical series of action of protein complexes at the DSB sites [7]. These proteins are primarily involved in protein-DNA and/or protein-protein interactions. Unfortunately, these interaction interfaces are challenging targets for small molecule inhibitors, which has significantly hindered development of potent and direct inhibitors of the HR pathway. In addition to small molecules that target the MRN complex (e.g., mirin [8], PFM01 and PFM39 [9]), chemical inhibitors of Rad51, the DNA recombinase involved in the final step of HR have also been identified [10]. However, most of these inhibitors suffer from low potency and/or selectivity, reflecting the challenges in directly drugging these protein complexes. Therefore, extensive efforts are ongoing to identify regulatory proteins of the HR pathway with the goal of indirectly drugging the HR pathway.

\section{Transcriptional regulation}

Bromodomain-containing proteins can recognize acetylated lysine residues in histone and nonhistone proteins to regulate transcription and protein-protein interactions [11]. These proteins are found to be highly amenable for small molecules and numerous chemical inhibitors have been developed to target a number of bromodomains. Among these, inhibitors of BRD4 were recently shown to potently inhibit HR efficiency in many different types of cancer cells, including breast cancer, ovarian cancer and pancreatic cancer [12,13]. Different mechanisms have been proposed for how the BRD4 inhibitors inhibit HR. One mechanism involves the transcription repression of BRCA1 and Rad51 [12] by BRD4 inhibitors, while another is through transcription inhibition of CtIP [13], whose gene product enhances the MRN complex's nuclease activity to process the DSB sites to initiate HR. It is possible that different chromatin states in different cell types may favor one mechanism over the other.

\section{Ubiquitination}

Another class of enzymes that have recently emerged as druggable targets to modulate the HR pathway is deubiqutinating enzymes (DUBs). Dynamic ubiquitination and deubiquitination of FANCD2, a protein component in the Fanconi anemia (FA) complex is required for proper function of the FA complex to promote HR [14]. USP1 is responsible for deubiquitinating FANCD2 and potent small molecule inhibitors (e.g., ML323) of USP1 have been developed [15]. These inhibitors have been shown to be able to suppress HR activity and resensitize cisplatin-resistant cancer cells to cisplatin [15]. UCHL3 is another DUB found to deubiquitinate Rad51 to promote its interaction with BRCA2 for loading to the processed DSB sites [16]. It will be interesting to see if potent and specific inhibitors of UCHL3 can be developed to sensitize the HR-proficient cells to PARP inhibitors or other DNA-damaging agents.

\section{Future perspective}

While the roles of USP1 and UCHL3 mentioned above are not necessarily related to their substrates degradation, other DUBs may be identified as important regulators of HR protein turnover. It is anticipated that inhibitors of these DUBs will likely impact HR by modulating the protein abundance. Along this same line of protein homeostasis, recently developed fragment-based screening strategies can be used to identify small molecule fragments that bind directly to HR proteins. Once these fragments are identified, they can be conjugated with a ubiquitin E3 ligase ligand (e.g., thalidomide) to create a proteolysis targeted chimera (PROTAC) for targeted protein degradation [17]. In conclusion, a better fundamental understanding of the regulation of the HR pathway and recent technology developments in designing inhibitors should facilitate rapid development of novel HR inhibitors as potential novel cancer therapies [18].

\section{Financial \& competing interests disclosure}

The authors are grateful for the financial support provided by the NIH (R01CA211866, R01GM122820 and R21CA220061) and the Elsa U Pardee Foundation. The authors have no other relevant affiliations or financial involvement with any organization or entity with a financial interest in or financial conflict with the subject matter or materials discussed in the manuscript apart from those disclosed.

No writing assistance was utilized in the production of this manuscript. 


\section{References}

1. Hanahan D, Weinberg RA. Hallmarks of cancer: the next generation. Cell 144(5), 646-674 (2011).

2. Chapman JR, Taylor MR, Boulton SJ. Playing the end game: DNA double-strand break repair pathway choice. Mol. Cell 47(4), 497-510 (2012).

3. Farmer H, McCabe N, Lord CJ et al. Targeting the DNA repair defect in BRCA mutant cells as a therapeutic strategy. Nature 434(7035), 917-921 (2005).

4. Halazonetis TD, Gorgoulis VG, Bartek J. An oncogene-induced DNA damage model for cancer development. Science 319(5868), 1352-1355 (2008).

5. Sakai W, Swisher EM, Karlan BY et al. Secondary mutations as a mechanism of cisplatin resistance in BRCA2-mutated cancers. Nature 451(7182), 1116-1120 (2008).

6. Norquist B, Wurz KA, Pennil CC et al. Secondary somatic mutations restoring BRCA1/2 predict chemotherapy resistance in hereditary ovarian carcinomas. J. Clin. Oncol. 29(22), 3008-3015 (2011).

7. Prakash R, Zhang Y, Feng W, Jasin M. Homologous recombination and human health: the roles of BRCA1, BRCA2, and associated proteins. Cold Spring Harb. Perspect. Biol. 7(4), a016600 (2015).

8. Dupre A, Boyer-Chatenet L, Sattler RM et al. A forward chemical genetic screen reveals an inhibitor of the Mre11-Rad50-Nbs1 complex. Nat. Chem. Biol. 4(2), 119-125 (2008).

9. Shibata A, Moiani D, Arvai AS et al. DNA double-strand break repair pathway choice is directed by distinct MRE11 nuclease activities. Mol. Cell 53(1), 7-18 (2014).

10. Budke B, Lv W, Kozikowski AP, Connell PP. Recent developments using small molecules to target RAD51: how to best modulate RAD51 for anticancer therapy? ChemMedChem 11(22), 2468-2473 (2016).

11. Fujisawa T, Filippakopoulos P. Functions of bromodomain-containing proteins and their roles in homeostasis and cancer. Nat. Rev. Mol. Cell Biol. 18(4), 246-262 (2017).

12. Yang L, Zhang Y, Shan W et al. Repression of BET activity sensitizes homologous recombination-proficient cancers to PARP inhibition. Sci. Transl. Med. 9(400), eaal1645 (2017).

13. Sun C, Yin J, Fang Y et al. BRD4 inhibition is synthetic lethal with PARP inhibitors through the induction of homologous recombination deficiency. Cancer Cell 33(3), 401-416. (2018).

14. Kee Y, D’Andrea AD. Expanded roles of the Fanconi anemia pathway in preserving genomic stability. Genes Dev. 24(16), 1680-1694 (2010).

15. Liang Q, Dexheimer TS, Zhang P et al. A selective USP1-UAF1 inhibitor links deubiquitination to DNA damage responses. Nat. Chem. Biol. 10(4), 298-304 (2014).

16. Luo $\mathrm{K}$, Li L, Li Y et al. A phosphorylation-deubiquitination cascade regulates the BRCA2-RAD51 axis in homologous recombination. Genes Dev. 30(23), 2581-2595 (2016).

17. Cromm PM, Crews CM. Targeted protein degradation: from chemical biology to drug discovery. Cell Chem. Biol. 24(9), 1181-1190 (2017).

18. Li BX, Chen J, Chao BAnticancer pyrroloquinazoline LBL1 targets nuclear lamins. ACS Chem. Biol. 13(5), 1380-1387 (2018). 
